Pacific

Journal of

Mathematics

INVARIANTS OF SOME COMPACTIFIED PICARD MODULAR SURFACES AND APPLICATIONS

AMIR DŽAMBIĆ

Volume $283 \quad$ No. 2

August 2016 


\title{
INVARIANTS OF SOME COMPACTIFIED PICARD MODULAR SURFACES AND APPLICATIONS
}

\author{
AMIR DŽAMBIĆ
}

\begin{abstract}
We investigate certain numerical invariants of compactified Picard modular surfaces by principal congruence subgroups of Picard modular groups. Applications to surface classification and modular forms are discussed.
\end{abstract}

\section{Introduction}

This article should be considered as a supplement to the work of R.-P. Holzapfel on invariants of Picard modular surfaces which are quotients of the two-dimensional complex ball by principal congruence subgroups.

Holzapfel [1980; 1998b] develops concrete formulas for the Chern numbers and related invariants of compactified ball quotients by principal congruence subgroups $\Gamma_{K(N)}$ of Picard modular groups $\Gamma_{K}=\operatorname{SU}\left((2,1), \mathscr{O}_{K}\right)$, where $K=\mathbb{Q}(\sqrt{-d})$ is an imaginary quadratic field and $N$ is a positive integer. There, he uses results which are mainly developed in [Holzapfel 1998a]. Using Riemann-Roch theory in combination with proportionality theorems, he also gets information on the classification of these compactified ball quotients and dimensions of spaces of cusp forms relative to the congruence subgroups.

In this article we slightly extend Holzapfel's results, considering not only principal congruence subgroups by natural numbers, but also general integral ideals $\mathfrak{a}$ of the quadratic field $K=\mathbb{Q}(\sqrt{-d})$. Including some technical, number theoretical details, Holzapfel's arguments can also be applied to this slightly more general and larger class of congruence subgroups. One technical result is a formula for the index $\left[\Gamma_{K}: \Gamma_{K}(\mathfrak{a})\right]$. The final results in principle do not differ from those obtained in [Holzapfel 1980;1998b]. We characterize the class of principal congruence subgroups - excluding on technical grounds a few possible "exceptional cases" for which the (smooth) compactified ball quotient is a surface of general type. For these surfaces the "coordinates" $\left(c_{2}, c_{1}^{2}\right)$ in the surface geography are explicitly known. The dimensions of spaces of cusp forms can also be computed explicitly. Besides the application to the geography of surfaces of general type let us also mention the very recent application of this geometric property in arithmetic geometry,

MSC2010: 11F06, 14G35, 14J29.

Keywords: Picard modular surfaces, surfaces of general type, congruence subgroups. 
as an ingredient in the proof of the mordellicity of the compactified congruence Picard modular surfaces by Dimitrov-Ramakrishnan [2015].

\section{Picard modular groups and their congruence subgroups}

Let $K=\mathbb{Q}(\sqrt{-d})$ be an imaginary quadratic field, and denote by $\mathscr{O}_{K}$ the ring of integers of $K$. Let $V$ be a three-dimensional $K$-vector space equipped with a hermitian form $h: V \times V \rightarrow K$. Let us assume that the signature of $h$ is $(2,1)$, i.e., that $h$ has two positive eigenvalues and one negative eigenvalue. Such $h$ can, for instance, be represented by the diagonal matrix $\operatorname{diag}(1,1,-1)$, but often it is useful to use another hermitian form. We consider the special unitary group

$$
\boldsymbol{G}:=\mathrm{SU}(h)=\left\{g \in \mathrm{SL}_{3}(\mathbb{C}) \mid h(g v, g w)=h(v, w) \text { for all } v, w \in V_{\mathbb{C}}\right\}
$$

as an algebraic group defined over $\mathbb{Q}$. Its group of $\mathbb{Q}$-rational points is

$$
\boldsymbol{G}(\mathbb{Q}):=\mathrm{SU}(h, K)=\left\{g \in \mathrm{SL}_{3}(K) \mid h(g v, g w)=h(v, w) \text { for all } w \in V\right\} .
$$

Often the interpretation of $\boldsymbol{G}$ as a group corresponding to an involution is useful. For this, we remark that the map $\iota=\iota_{h}$, which associates with each matrix $g \in M_{3}(K)$ the matrix $g^{l_{h}}:=M_{h} \bar{g}^{t} M_{h}^{-1}$, where $M_{h}$ denotes the matrix which represents $h$ with respect to a suitable basis $(\operatorname{diag}(1,1,-1)$, say), defines an involution of the second kind on the matrix algebra $M_{3}(K)$. This means that $\iota$ is an antiautomorphism of $M_{3}(K)$ which acts as the complex conjugation, when restricted to the diagonal matrices $\operatorname{diag}(\alpha, \alpha, \alpha)$. Under this interpretation, the group $\boldsymbol{G}$ appears as the group of all matrices $g \in \mathrm{SL}_{3}$ such that $g g^{l_{h}}=1_{3}$.

We define the so-called full Picard modular group as

$$
\Gamma_{K}=\mathrm{SU}\left(h, \mathscr{O}_{K}\right)=\mathrm{SL}_{3}\left(\mathscr{O}_{K}\right) \cap \boldsymbol{G}(\mathbb{Q}) .
$$

$\Gamma_{K}$ is obviously an arithmetic subgroup in $\boldsymbol{G}(\mathbb{Q})$ as well as its subgroups of finite index, which we simply call Picard modular groups. If $\mathfrak{a}$ is an ideal of $\mathfrak{O}_{K}$, let $\Gamma_{K}(\mathfrak{a})$ denote the principal congruence subgroup of $\Gamma_{K}$ with respect to $\mathfrak{a}$. It is defined as the subgroup of $\Gamma_{K}$ consisting of all elements $\gamma \in \Gamma_{K}$ such that $\gamma-1_{3} \in M_{3}(\mathfrak{a})$. In other words, $\Gamma_{K}(\mathfrak{a})$ can be defined as the kernel of the canonical reduction map

$$
\rho: \Gamma_{K} \rightarrow \mathrm{SL}_{3}\left(\mathrm{O}_{K} / \mathfrak{a}\right) .
$$

We define the level of $\Gamma_{K}(\mathfrak{a})$ as the absolute norm $N(\mathfrak{a})=\left|O_{K} / \mathfrak{a}\right|$. There are finitely many principal congruence subgroups of fixed level. By definition, a congruence subgroup of $\Gamma_{K}$ is a group which contains a principal congruence subgroup. Since every principal congruence subgroup has a finite index in $\Gamma_{K}$ (which is obvious by definition), all congruence subgroups are Picard modular groups. In this section we will treat the following two technical problems: 
- computing the index $\left[\Gamma_{K}: \Gamma_{K}(\mathfrak{a})\right]$,

- determining $\mathfrak{a}$ for which the principal congruence subgroup $\Gamma_{K}(\mathfrak{a})$ is a neat subgroup.

Note that we say that an arithmetic group is neat if the subgroup of $\mathbb{C}^{*}$ generated by the eigenvalues of all elements in $\Gamma$ is torsion free. A neat group is obviously torsion free.

Index computations. In order to compute the index $\left[\Gamma_{K}: \Gamma_{K}(\mathfrak{a})\right]$, we make use of the local-to-global principle which is applicable in the present case.

For a prime number $p$ the group of $\mathbb{Q}_{p}$-rational points $\boldsymbol{G}\left(\mathbb{Q}_{p}\right)$ is

$$
\boldsymbol{G}\left(\mathbb{Q}_{p}\right)=\left\{g \in \mathrm{SL}_{3}\left(K \otimes_{\mathbb{Q}} \mathbb{Q}_{p}\right) \mid g g^{l_{p}}=1_{3}\right\},
$$

where we write $\iota_{p}$ for the natural extension of $\iota_{h}$ to the algebra $M_{3}\left(K \otimes_{\mathbb{Q}} \mathbb{Q}_{p}\right)$. The group $\boldsymbol{G}\left(\mathbb{Z}_{p}\right)$ is defined in an obvious manner. We can also define principal congruence subgroups $\boldsymbol{G}\left(\mathbb{Z}_{p}\right)(\mathfrak{a})$ of $\boldsymbol{G}\left(\mathbb{Z}_{p}\right)$ in the same way as in the case of $\Gamma_{K}$, now taking two-sided ideals $\mathfrak{a}$ of the order $\mathrm{O}_{K} \otimes \mathbb{Z}_{p}$ into account. The following lemma is the starting point of the index calculation. It is an important consequence of strong approximation.

Lemma 2.1. Let $\mathfrak{a} \subset \mathfrak{O}_{K}$ be an integral ideal of $K$, let $\mathfrak{a}=\mathfrak{p}_{1}^{e_{1}} \cdots \mathfrak{p}_{t}^{e_{t}}$ be the prime ideal decomposition of $\mathfrak{a}$, and denote by $p_{i}=\mathfrak{p}_{i} \cap \mathbb{Z}$ the unique integer prime over which $\mathfrak{p}$ lies. Then there is an isomorphism

$$
\Gamma_{K} / \Gamma_{K}(\mathfrak{a}) \cong \prod_{i=1}^{t} \boldsymbol{G}\left(\mathbb{Z}_{p_{i}}\right) / \boldsymbol{G}\left(\mathbb{Z}_{p_{i}}\right)\left(\mathfrak{p}^{e_{i}}\right) .
$$

In particular,

$$
\left[\Gamma_{K}: \Gamma_{K}(\mathfrak{a})\right]=\prod_{i=1}^{t}\left[\boldsymbol{G}\left(\mathbb{Z}_{p_{i}}\right): \boldsymbol{G}\left(\mathbb{Z}_{p_{i}}\right)\left(\mathfrak{p}^{e_{i}}\right)\right] .
$$

Proof. Let $\lambda$ be the map

$$
\lambda: \Gamma_{K} \longrightarrow \prod_{i=1}^{t} \boldsymbol{G}\left(\mathbb{Z}_{p_{i}}\right) / \boldsymbol{G}\left(\mathbb{Z}_{p_{i}}\right)\left(\mathfrak{p}^{e_{i}}\right)
$$

given by

$$
\gamma \mapsto\left(\gamma \bmod \mathfrak{p}_{1}^{e_{1}}, \ldots, \gamma \bmod \mathfrak{p}_{t}^{e_{t}}\right)
$$

The kernel of $\lambda$ is obviously $\Gamma_{K}(\mathfrak{a})$, by definition. To show surjectivity we recall the strong approximation property: for any finite set of primes $p_{1}, \ldots, p_{t}$ and elements $g_{p_{1}} \in \boldsymbol{G}\left(\mathbb{Z}_{p_{1}}\right), \ldots, g_{p_{t}} \in \boldsymbol{G}\left(\mathbb{Z}_{p_{t}}\right)$ as well as exponents $e_{1}, \ldots, e_{t}$, one can find an element $g \in \boldsymbol{G}(\mathbb{Z})=\Gamma_{K}$ such that $g \equiv g_{p_{i}} \bmod p_{i}^{e_{i}}$ for $i=1, \ldots, t$. But as $\mathfrak{p}_{i} \mid p_{i}$ this $g$ satisfies $g \equiv g_{p_{i}} \bmod \mathfrak{p}_{i}^{e_{i}}$. This proves the surjectivity of $\lambda$. 
In order to compute the local indices, we first need to know more about the local groups $\boldsymbol{G}\left(\mathbb{Q}_{p}\right)$. Looking at their definition (2-1), we see that their structure highly depends on the structure of $K \otimes \mathbb{Q}_{p}$, which itself is determined by the decomposition behavior of the prime $p$. Essentially there are two cases:

(i) $p$ is decomposed in $K=\mathbb{Q}(\sqrt{-d})$. In this case $K \otimes \mathbb{Q}_{p} \cong K_{\mathfrak{p}} \oplus K_{\overline{\mathfrak{p}}}$ with two conjugate prime ideals $\mathfrak{p} \neq \overline{\mathfrak{p}}$ in $\mathscr{O}_{K}$ such that $p=\mathfrak{p} \overline{\mathfrak{p}}$. Therefore

$$
M_{3}\left(K \otimes \mathbb{Q}_{p}\right) \cong M_{3}\left(K_{\mathfrak{p}}\right) \oplus M_{3}\left(K_{\overline{\mathfrak{p}}}\right) .
$$

Since $p$ is decomposed in $K,\left(\frac{-d}{p}\right)=1$ and $-d$ is a square in $\mathbb{Q}_{p}$. Therefore $K_{\mathfrak{p}} \cong K_{\overline{\mathfrak{p}}} \cong \mathbb{Q}_{p}$. The extension of the field automorphism ${ }^{-}: K \rightarrow K$ to $K \otimes \mathbb{Q}_{p}$ must be an involution on $K_{\mathfrak{p}} \oplus K_{\overline{\mathfrak{p}}} \cong \mathbb{Q}_{p} \oplus \mathbb{Q}_{p}$ and $\overline{(x, y)}=(y, x)$ is the only possibility. Therefore the extension $\iota_{p}$ of $\iota=\iota_{h}$ is given by changing the summands in $M_{3}\left(\mathbb{Q}_{p}\right) \oplus M_{3}\left(\mathbb{Q}_{p}\right)$. Now it is easy to see that the projection on one of the summands gives an isomorphism between $\boldsymbol{G}\left(\mathbb{Q}_{p}\right)$ and $\mathrm{SL}_{3}\left(\mathbb{Q}_{p}\right)$, since $\boldsymbol{G}\left(\mathbb{Q}_{p}\right)$ is defined as the group consisting of those pairs $(g, h) \in \mathrm{SL}_{3}\left(\mathbb{Q}_{p}\right) \oplus \mathrm{SL}_{3}\left(\mathbb{Q}_{p}\right)$ with $\left(g^{-1}, h^{-1}\right)=(h, g)$.

(ii) $p$ is nondecomposed in $K$. In this case there is a unique prime ideal $\mathfrak{p}$ lying over $p$, such that $K \otimes \mathbb{Q}_{p}=K_{\mathfrak{p}}$, which is a quadratic extension of $\mathbb{Q}_{p}$. The extended involution $\iota_{p}$ is an involution of the second kind on $M_{3}\left(K_{\mathfrak{p}}\right)$ given by $g \mapsto M_{h} \bar{g} M_{h}^{-1}$ and $\boldsymbol{G}\left(\mathbb{Q}_{p}\right)=\mathrm{SU}\left(h, K_{\mathfrak{p}}\right)$.

Lemma 2.2. If $p$ is nondecomposed, $\boldsymbol{G}\left(\mathbb{Q}_{p}\right) \cong \mathrm{SU}_{3}\left(K_{\mathfrak{p}}\right)$.

Proof. By Landherr's theorem, the isometry class of a hermitian form $h$ in $n$ variables associated with a quadratic extension $E / F$ of local fields is uniquely determined by its discriminant $\boldsymbol{d}(h)=[\operatorname{det}(h)] \in F^{*} / N_{E / F}\left(E^{*}\right) \cong \mathbb{Z} / 2 \mathbb{Z}$. On the other hand, the isomorphism class of the associated unitary group $\mathrm{SU}(h)$ only depends on $h$ up to a multiplicative constant. Hence, the unitary group associated with $\operatorname{diag}(1,1,-1)$ is isomorphic to the unitary group associated with $\operatorname{diag}(-1,-1,1)$. Since the discriminant of the latter is 1 , the result follows.

Now we compute the local indices:

Lemma 2.3. Let $\mathfrak{p}$ be a prime ideal of $K=\mathbb{Q}(\sqrt{-d})$ such that $p=\mathfrak{p} \cap \mathbb{Z}$ is decomposed in $K$. Then

$$
\left[\boldsymbol{G}\left(\mathbb{Z}_{p}\right): \boldsymbol{G}\left(\mathbb{Z}_{p}\right)\left(\mathfrak{p}^{n}\right)\right]=p^{8 n}\left(1-p^{-3}\right)\left(1-p^{-2}\right)
$$

for any positive integer $n$.

Proof. By our discussion above, in the decomposed case $\boldsymbol{G}\left(\mathbb{Z}_{p}\right)=\mathrm{SL}_{3}\left(\mathbb{Z}_{p}\right)$ and $\boldsymbol{G}\left(\mathbb{Z}_{p}\right)\left(\mathfrak{p}^{n}\right)=\mathrm{SL}_{3}\left(\mathbb{Z}_{p}\right)\left(p^{n}\right)$. We note that the sequence

$$
1 \longrightarrow \mathrm{SL}_{n}\left(\mathbb{Z}_{p}\right)\left(p^{n}\right) \longrightarrow \mathrm{SL}_{n}\left(\mathbb{Z}_{p}\right) \longrightarrow \mathrm{SL}_{n}\left(\mathbb{Z}_{p} / p^{n} \mathbb{Z}_{p}\right) \longrightarrow 1
$$


is exact. In fact, (2-2) is exact even if we replace $\mathbb{Z}_{p}$ and $p^{n}$ by any commutative ring $R$ and ideal $\mathfrak{I}$ such that $R / \mathfrak{I}$ is finite (see [Bass 1964], Corollary 5.2). Therefore

$$
\left[\boldsymbol{G}\left(\mathbb{Z}_{p}\right): \boldsymbol{G}\left(\mathbb{Z}_{p}\right)\left(\mathfrak{p}^{n}\right)\right]=\left|\mathrm{SL}_{3}\left(\mathbb{Z}_{p} / p^{n} \mathbb{Z}_{p}\right)\right|=\left|\mathrm{SL}_{3}\left(\mathbb{Z} / p^{n} \mathbb{Z}\right)\right|
$$

The latter number can be computed in an elementary way: Let $\rho: \mathrm{GL}_{3}\left(\mathbb{Z} / p^{n} \mathbb{Z}\right) \rightarrow$ $\mathrm{GL}_{3}(\mathbb{Z} / p \mathbb{Z})$ be the canonical projection map which sends every residue $\bmod p^{n}$ to its residue $\bmod p$. The kernel $\operatorname{ker}(\rho)$ consists of $p^{9(n-1)}$ elements. With the well-known number $\left|\mathrm{GL}_{3}(\mathbb{Z} / p \mathbb{Z})\right|=p^{9}\left(1-p^{-3}\right)\left(1-p^{-2}\right)\left(1-p^{-1}\right)$, we get $\left|\mathrm{GL}_{3}\left(\mathbb{Z} / p^{n} \mathbb{Z}\right)\right|=p^{9 n}\left(1-p^{-3}\right)\left(1-p^{-2}\right)\left(1-p^{-1}\right)$. Dividing this number by $\varphi\left(p^{n}\right)$, which is the order of the multiplicative group $\left(\mathbb{Z} / p^{n} \mathbb{Z}\right)^{*}$, we get the desired result.

For some technical reasons, from now on we exclude from consideration the prime 2 .

Lemma 2.4. Let $\mathfrak{p}$ be a prime ideal in $\mathfrak{O}_{\boldsymbol{K}}$ and $p=\mathfrak{p} \cap \mathbb{Z} \neq 2$ nondecomposed. For every $n \geq 1$ the following hold:

(1) If $p$ is inert,

$$
\left[\boldsymbol{G}\left(\mathbb{Z}_{p}\right): \boldsymbol{G}\left(\mathbb{Z}_{p}\right)\left(\mathfrak{p}^{n}\right)\right]=p^{8 n}\left(1+p^{-3}\right)\left(1-p^{-2}\right) .
$$

(2) If $p$ is ramified,

$$
\left[\boldsymbol{G}\left(\mathbb{Z}_{p}\right): \boldsymbol{G}\left(\mathbb{Z}_{p}\right)\left(\mathfrak{p}^{n}\right)\right]=p^{\epsilon_{n}}\left(1-p^{-2}\right),
$$

where $\epsilon_{n}$ is defined as

$$
\epsilon_{n}=\left\{\begin{array}{cc}
4 n & \text { if } n \equiv 0 \bmod 2, \\
4 n-1 & \text { if } n \equiv 1 \bmod 2 .
\end{array}\right.
$$

Proof. First, we identify the desired index with the order of the finite group $\operatorname{SU}\left(\bar{h}, \mathscr{O}_{\mathfrak{p}} / \mathfrak{p}^{n}\right)$, where $\mathscr{O}_{\mathfrak{p}}=\mathscr{O}_{K_{\mathfrak{p}}}$ denotes the ring of integers in $K_{\mathfrak{p}}$ and $\bar{h}$ the restriction of $h$ to $O_{\mathfrak{p}} / \mathfrak{p}^{n}$. To do so, we have to know that the canonical reduction $\operatorname{map} \boldsymbol{G}\left(\mathbb{Z}_{p}\right)=\mathrm{SU}\left(h, \mathscr{O}_{\mathfrak{p}}\right) \rightarrow \mathrm{SU}\left(\bar{h}, \mathscr{O}_{\mathfrak{p}} / \mathfrak{p}^{n}\right)$ is surjective. But this is a rather general fact (see for instance [Baeza 1973, Bemerkung (4.3)]). Moreover, we can extract the above local index formulas again from [Holzapfel 1998a, Corollary 5A.1.3] for the case in which $p$ is inert, or $p$ is ramified and $n$ is an even positive integer. According to this, the only nontrivial case is the situation where $p$ is ramified and $n$ is odd. Let us from now on assume this situation, that is, $p$ is ramified and $n$ is odd.

By [Holzapfel 1998a, Corollary 5A.1.3] again we know that

$$
\boldsymbol{G}\left(\mathbb{Z}_{p}\right) / \boldsymbol{G}\left(\mathbb{Z}_{p}\right)\left(\mathfrak{p}^{n+1}\right)=\left|\boldsymbol{G}\left(\mathbb{Z}_{p}\right) / \boldsymbol{G}\left(\mathbb{Z}_{p}\right)\left(p^{(n+1) / 2}\right)\right|=p^{4(n+1)}\left(1-p^{-2}\right) .
$$

By the multiplicativity of the index we obtain

$$
\boldsymbol{G}\left(\mathbb{Z}_{p}\right) / \boldsymbol{G}\left(\mathbb{Z}_{p}\right)\left(\mathfrak{p}^{n}\right)=p^{4(n+1)}\left(1-p^{-2}\right) /\left[\boldsymbol{G}\left(\mathbb{Z}_{p}\right)\left(\mathfrak{p}^{n}\right) / \boldsymbol{G}\left(\mathbb{Z}_{p}\right)\left(\mathfrak{p}^{n+1}\right)\right] .
$$


In order to compute the index in the denominator, we first recall the isomorphism $\boldsymbol{G}\left(\mathbb{Q}_{p}\right) \cong \mathrm{SU}_{3}\left(K_{\mathfrak{p}}\right)$ from Lemma 2.2. Let $\pi$ be a generator of the valuation ideal $\mathfrak{p}$ of $O_{\mathfrak{p}}$. As $p \neq 2$, the extension $K_{\mathfrak{p}} / \mathbb{Q}_{p}$ is tamely ramified and we can choose $\pi$ such that $\bar{\pi}=-\pi$, where ${ }^{-}$denotes the conjugation in $K_{\mathfrak{p}}$ (compare [Lang 1970, Proposition II.5.12]).

As a matrix in $M_{3}\left(K_{\mathfrak{p}}\right)$, every $g \in \boldsymbol{G}\left(\mathbb{Z}_{p}\right)$ has a $\mathfrak{p}$-adic representation:

$$
g=g_{0}+\pi g_{1}+\pi^{2} g_{2}+\pi^{3} g_{3}+\cdots,
$$

for some matrices $g_{j} \in M_{3}\left(O_{\mathfrak{p}} / \mathfrak{p}\right)=M_{3}(\mathbb{Z} / p \mathbb{Z})$, where the latter equality holds since $p$ is ramified. We note that a matrix $g \in \boldsymbol{G}\left(\mathbb{Z}_{p}\right)$ lies in $\boldsymbol{G}\left(\mathbb{Z}_{p}\right)\left(\mathfrak{p}^{n}\right)$ if and only if its $\mathfrak{p}$-adic representation starts with

$$
g=1_{3}+\pi^{n} g_{n}+\pi^{n+1} g_{n+1}+\cdots .
$$

For this reason, the map $g \mapsto g_{n}$ gives a map

$$
\boldsymbol{G}\left(\mathbb{Z}_{p}\right) / \boldsymbol{G}\left(\mathbb{Z}_{p}\right)\left(\mathfrak{p}^{n}\right) \longrightarrow M_{3}(\mathbb{Z} / p \mathbb{Z}) .
$$

Note that, as $p$ is ramified, the conjugation acts as the identity on $O_{\mathfrak{p}} / \mathfrak{p}^{n}$. Consider a representative $g \in \boldsymbol{G}\left(\mathbb{Z}_{p}\right)\left(\mathfrak{p}^{n}\right)$ of the class $[g] \in \boldsymbol{G}\left(\mathbb{Z}_{p}\right)\left(\mathfrak{p}^{n}\right) / \boldsymbol{G}\left(\mathbb{Z}_{p}\right)\left(\mathfrak{p}^{n+1}\right)$. Two equations characterize $g$ as an element of $\mathrm{SU}_{3}\left(K_{\mathfrak{p}}\right)$. First, $g$ is hermitian, that is,

$$
g \bar{g}^{t}=\left(1_{3}+\pi^{n} g_{n}+\cdots\right)\left(1_{3}+\bar{\pi}^{n} \bar{g}_{n}+\cdots\right)^{t}=1_{3}
$$

or, equivalently,

$$
\left(1_{3}+\pi^{n} g_{n}+\cdots\right)\left(1_{3}-\pi^{n} \bar{g}_{n}^{t}+\cdots\right)=1_{3} .
$$

In other words, $g_{n}-g_{n}^{t}=0$, that is, $g_{n}$ is symmetric. On the other hand, as

$$
\operatorname{det}(g)=\operatorname{det}\left(1_{3}+\pi^{n} g_{n}+\cdots\right)=1 \equiv 1+\pi^{n} \operatorname{Tr}\left(g_{n}\right) \bmod \pi^{n+1},
$$

$g_{n}$ has trace 0 . By this, the factor group $G\left(\mathbb{Z}_{p}\right)\left(\mathfrak{p}^{n}\right) / \boldsymbol{G}\left(\mathbb{Z}_{p}\right)\left(\mathfrak{p}^{n+1}\right)$ is characterized as the set of symmetric matrices in $M_{3}(\mathbb{Z} / p \mathbb{Z})$ with trace equal to 0 . There are exactly $p^{5}$ such matrices, and (2-3) gives for $n$ odd and $p$ ramified

$$
\boldsymbol{G}\left(\mathbb{Z}_{p}\right) / \boldsymbol{G}\left(\mathbb{Z}_{p}\right)\left(\mathfrak{p}^{n}\right)=p^{4 n-1}\left(1-p^{-2}\right),
$$

from which the final formula follows.

Now, summarizing results from Lemma 2.3 and Lemma 2.4, we get:

Theorem 2.5. Let $\mathfrak{a}$ be an integral ideal in $K=\mathbb{Q}(\sqrt{-d})$ and let $\mathfrak{a}=\mathfrak{p}_{1}^{e_{1}} \cdots \mathfrak{p}_{t}^{e_{t}}$ be the prime ideal decomposition of $\mathfrak{a}$ with $p_{i}=\mathfrak{p}_{i} \cap \mathbb{Z}$. Assume that $p_{i} \neq 2$ for all $i=1, \ldots, t$, if 2 is nondecomposed in $K$. Then it holds that

$$
\left[\Gamma_{K}: \Gamma_{K}(\mathfrak{a})\right]=\prod_{i=1}^{t} p^{\epsilon_{i}}\left(1-p_{i}^{-2}\right)\left(1-\chi_{D}\left(p_{i}\right) p_{i}^{-3}\right),
$$


where $D$ is the discriminant of $K, \chi_{D}()$ is the Jacobi symbol $\chi_{D}(p)=\left(\frac{D}{p}\right)$ and $\epsilon_{i}$ is defined by

$$
\epsilon_{i}=\left\{\begin{array}{cl}
8 e_{i} & \text { if } \chi_{D}\left(p_{i}\right) \neq 0, \\
4 e_{i} & \text { if } \chi_{D}\left(p_{i}\right)=0 \text { and } e_{i} \equiv 0 \bmod 2, \\
4 e_{i}-1 & \text { if } \chi_{D}\left(p_{i}\right)=0 \text { and } e_{i} \equiv 1 \bmod 2 .
\end{array}\right.
$$

We remark that this result generalizes [Holzapfel 1998a, Theorem 5A.2.14].

Determination of neat subgroups. In this section we discuss the question, for which integral ideals $\mathfrak{a}$ the principal congruence subgroup $\Gamma_{K}(\mathfrak{a})$ is a neat subgroup. This rather technical property will be used in our consideration of Picard modular surfaces.

Lemma 2.6. Assume that $\mathfrak{a}$ is an ideal in $\mathrm{O}_{K}$ such that $\mathfrak{a} \cap \mathbb{Z}$ and 2 are coprime. Then the principal congruence subgroup $\Gamma_{K}(\mathfrak{a})$ is neat if $N(\mathfrak{a})>3$.

Proof. (Compare [Holzapfel 1980, Lemma 4.3].) Assume that $\Gamma_{K}(\mathfrak{a})$ is not neat. Then there are eigenvalues $\zeta$ of elements $\gamma \in \Gamma_{K}(\mathfrak{a})$ which generate a torsion subgroup in $\mathbb{C}^{*}$. One concludes that such eigenvalues must be roots of unity [Holzapfel 1980, proof of Lemma 4.3]. Now, on the one hand, such an eigenvalue $\zeta$ is a root of unity, $n$-th root of unity, say, and on the other hand, $\zeta$ is a zero of the characteristic polynomial of $\gamma$ which has degree 3 over $K$. Therefore $[\mathbb{Q}(\zeta): \mathbb{Q}]=\varphi(n) \leq 6$. Without loss of generality we can assume that $n=p$ is a prime number. So, in fact we have to check that for $n=2,3,5,7$ the primitive $n$-th root of unity does not appear as the eigenvalue of elements in $\Gamma_{K}(\mathfrak{a})$ for stated ideals $\mathfrak{a}$. For this, we first observe that there is a relation between the eigenvalues $\zeta$ of elements in $\Gamma_{K}(\mathfrak{a})$ and the ideal $\mathfrak{a}$, namely

$$
\mathfrak{a} \mathbb{Z}[\zeta] \mid(\zeta-1) \mathbb{Z}[\zeta]
$$

Taking norms on both sides we obtain

$$
N(\mathfrak{a} \mathbb{Z}[\zeta])=N(\mathfrak{a})^{\varphi(n) / 2} \mid N_{\mathbb{Q}(\zeta) / \mathbb{Q}}(\zeta-1)=n .
$$

It is easily checked that none of the above primes satisfies this relation for given $\mathfrak{a}$ with $(2, \mathfrak{a} \cap \mathbb{Z})=1$ and $N(\mathfrak{a})>3$.

\section{Proportionality principle for compactified Picard modular surfaces}

As a noncompact almost simple Lie group, $\boldsymbol{G}(\mathbb{R})=\mathrm{SU}(h, \mathbb{C})$ has a naturally associated irreducible simply connected hermitian symmetric domain $\mathbb{B}=\mathrm{SU}(h) / C$, with a maximal compact subgroup $C$. Taking $h$ to be represented by $\operatorname{diag}(1,1,-1)$, this symmetric domain is exactly the two-dimensional complex unit ball

$$
\mathbb{B}=\mathbb{B}_{2}=\left\{\left.\left(z_{1}, z_{2}\right)|| z_{1}\right|^{2}+\left|z_{2}\right|^{2}<1\right\} .
$$


It is more natural to consider $\mathbb{B}$ embedded in $\mathbb{P}_{2}(\mathbb{C})$, which is interpreted as the compact dual symmetric space of $\mathbb{B}$. From the construction of $\mathbb{B}$, it is clear that the group $\mathrm{SU}(h)$ acts on $\mathbb{B}$ as a group of biholomorphic transformations. According to the theorem of Borel and Harish-Chandra (see [Borel and Harish-Chandra 1962]), the group $\Gamma_{K}$, and consequently every group $\Gamma$ commensurable to $\Gamma_{K}$, is a discrete subgroup of finite covolume in the Lie group $\boldsymbol{G}(\mathbb{R})=\mathrm{SU}(h, \mathbb{C})$ corresponding to $\boldsymbol{G}$. Therefore, the action of each $\Gamma$ is properly discontinuous, and it is reasonable to consider the quotient space $Y(\Gamma):=\Gamma \backslash \mathbb{B}$. Now we recall some basic properties of $\bar{Y}(\Gamma)$.

Theorem 3.1. Let $\Gamma \subset \Gamma_{K}$ be a Picard modular group and $Y(\Gamma)=\Gamma \backslash \mathbb{B}$ the corresponding locally symmetric space. Then the following hold:

(1) $Y(\Gamma)$ is not compact.

(2) There exists a compactification $\bar{Y}(\Gamma)$ of $Y(\Gamma)$ (the so-called Baily-Borel compactification), which has a structure of a normal projective surface.

(3) Let $\tilde{Y}(\Gamma)$ be the minimal resolution of the singularities of $\bar{Y}(\Gamma)$. We call, for brevity, $\widetilde{Y}(\Gamma)$ the smooth compactification of $Y(\Gamma)$.

The proofs of the above statements, which are in fact special cases of theorems that are valid in much greater generality, can be found in [Holzapfel 1998a]. Let us roughly sketch the main steps of the proofs, also in order to introduce some notions which will be used later. The noncompactness follows from [Borel and Harish-Chandra 1962, Theorem 11.8]. By that theorem, $Y(\Gamma)$ is compact if and only if there are no nontrivial unipotent elements in $\boldsymbol{G}(\mathbb{Q})$. But there are many unipotent elements and they all lie in the unipotent radicals of minimal rational parabolic subgroups of $\boldsymbol{G}$, which themselves fix maximal $h$-isotropic subspaces of $V=K^{3}$. The compactification $\bar{Y}(\Gamma)$ of $Y(\Gamma)$ is obtained by taking the quotient $\bar{Y}(\Gamma)=\Gamma \backslash \mathbb{B}^{*}$, where $\mathbb{B}^{*}=\mathbb{B} \cup \partial_{\mathbb{Q}} \mathbb{B}$ with $\partial_{\mathbb{Q}} \mathbb{B}=\boldsymbol{G}(\mathbb{Q}) / \boldsymbol{P}$ for some fixed rational parabolic subgroup $\boldsymbol{P} \subset \boldsymbol{G}$. By a suitable choice of $\boldsymbol{P}$ one can assume that $\partial_{\mathbb{Q}} \mathbb{B}=\left\{[v] \in \mathbb{P}_{2}(K) \mid h(v, v)=0\right\}$. The set $\partial_{\Gamma} \mathbb{B}=\Gamma \backslash \boldsymbol{G}(\mathbb{Q}) / \boldsymbol{P}$ is finite, so $\bar{Y}(\Gamma)=Y(\Gamma) \cup$ \{finitely many points $\}$. By the theorem of Baily-Borel, $\bar{Y}(\Gamma)$ is a normal projective variety. The singularities come from nontrivial torsion elements in $\Gamma$ and possibly from cusps, that is, points of $\bar{Y}(\Gamma)-Y(\Gamma)$. If we assume that $\Gamma$ is neat, the theory of toroidal compactifications (see [Holzapfel 1998a, Chapter 4] for the special case of arithmetic lattices in $\mathrm{SU}(2,1))$ provides a smooth compactification by replacing every cusp by a smooth elliptic curve. Note that, by a theorem of Borel, neat normal subgroups $\Gamma^{\prime} \triangleleft \Gamma_{K}$ of finite index exist. If $\Gamma$ is not neat, the toroidal compactification provides a surface with at most quotient singularities. They can be resolved by known methods. This resolution gives a map $\tilde{Y}(\Gamma) \rightarrow \bar{Y}(\Gamma)$, with smooth $\tilde{Y}(\Gamma)$. 
Chern numbers of compactified Picard modular surfaces. The two Chern numbers $c_{2}$ and $c_{1}^{2}$ of a smooth compact complex surface are important numerical invariants. The famous proportionality theorem of $\mathrm{F}$. Hirzebruch states that the Chern numbers of a (two-dimensional) ball quotient by a torsion free cocompact discrete subgroup satisfy $c_{1}^{2}=3 c_{2}$. D. Mumford generalized Hirzebruch's theorem also to noncompact quotients. Before we state the proportionality result for Picard modular groups, as described in [Holzapfel 1998a], we need to introduce some notions.

Let $\Gamma$ be a neat Picard modular group. The quotient $Y(\Gamma)$ is not compact, but one can still define Chern numbers of $Y(\Gamma)$ as the volumes

$$
c_{2}(Y(\Gamma))=\int_{F(\Gamma)} \eta_{2} \quad \text { and } \quad c_{1}^{2}(Y(\Gamma))=\int_{F(\Gamma)} \eta_{1}^{2},
$$

where $F(\Gamma)$ is a fundamental domain of $\Gamma$ in $\mathbb{B}$ and $\eta_{2}$ and $\eta_{1}^{2}$ are suitably normalized volume forms related to the $\Gamma$-invariant Bergman metric on $\mathbb{B}$.

On the other hand, on the smooth compactification $\tilde{Y}(\Gamma)$ of $Y(\Gamma)$ there are also the usual Chern numbers. Before we relate these two kinds of Chern numbers, we note that $\tilde{Y}(\Gamma)$ arises from $Y(\Gamma)$ by replacing a cusp $\kappa \in \partial_{\Gamma} \mathbb{B}$ by an elliptic curve $E_{\kappa}$. Hence, the difference between $\tilde{Y}(\Gamma)$ and $Y(\Gamma)$ is encoded in the compactification divisor $T_{\Gamma}=\sum_{\kappa \in \partial_{\Gamma} \mathbb{B}} E_{\kappa}$.

Proposition 3.2 [Holzapfel 1998a, Proposition 4.3.6]. Let $\Gamma$ be a neat Picard modular group, $Y(\Gamma)=\Gamma \backslash \mathbb{B}$ and $\tilde{Y}(\Gamma)$ be the smooth compactification of $Y(\Gamma)$. Also, let $T_{\Gamma}$ denote the compactification divisor and $\left(T_{\Gamma} . T_{\Gamma}\right)$ the self-intersection number of $T_{\Gamma}$. Then:

(1) $c_{2}(\tilde{Y}(\Gamma))=c_{2}(Y(\Gamma))$,

(2) $c_{1}^{2}(\tilde{Y}(\Gamma))=3 c_{2}(Y(\Gamma))+\left(T_{\Gamma} \cdot T_{\Gamma}\right)$.

Chern numbers of quotients by principal congruence subgroups. By the above Proposition 3.2, two magnitudes are essential for the computation of Chern invariants of the smooth compactification of a Picard modular surface $Y(\Gamma)=\Gamma \backslash \mathbb{B}$, namely the volume $c_{2}(Y(\Gamma))$ and the self-intersection number $\left(T_{\Gamma} \cdot T_{\Gamma}\right)$. In the case of a ball quotient by a principal congruence subgroup, these two numbers can be given in terms of a number theoretic expression. Let us for brevity write $Y(\mathfrak{a})$ for the ball quotient $\Gamma_{K}(\mathfrak{a}) \backslash \mathbb{B}$ by the principal congruence subgroup $\Gamma_{K}(\mathfrak{a})$. In this context we also write $Y(1)$ for the quotient $\Gamma_{K} \backslash \mathbb{B}$ by a full Picard modular group.

Proposition 3.3 (see [Holzapfel 1998a, Theorem 5A.4.7]). Let $K=\mathbb{Q}(\sqrt{-d})$ and let $\Gamma_{K}$ be the full Picard modular group corresponding to $K$. Let $D$ be the discriminant of $K$ and $\chi_{D}()=\left(\frac{D}{.}\right)$ the Dirichlet character associated with $K$. 
Then

$$
c_{2}(Y(1))=c_{2}\left(\Gamma_{K} \backslash \mathbb{B}\right)=\delta_{K} \frac{|D|^{5 / 2}}{32 \pi^{3}} L\left(3, \chi_{D}\right),
$$

where $L\left(s, \chi_{D}\right)$ denotes the Dirichlet $L$-function associated with $\chi_{D}$, and $\delta_{K}$ is the order of the center of $\Gamma_{K}$, that is, $\delta_{K}=\frac{1}{3}$ if $d=3$, and $\delta_{K}=1$ otherwise.

Let $\mathbb{P} \Gamma$ be the quotient of $\Gamma$ by its center. We already know $c_{2}(Y(\mathfrak{a}))=c_{2}(\tilde{Y}(\mathfrak{a}))$. As $\tilde{Y}(\mathfrak{a})$ is a smooth Galois covering of $\tilde{Y}(1)$ and $\left[\mathbb{P} \Gamma_{K}: \mathbb{P} \Gamma_{K}(\mathfrak{a})\right]=\left[\Gamma_{K}: \Gamma_{K}(\mathfrak{a})\right] / \delta_{K}$ is the degree of this covering, Proposition 3.3 implies the following result:

Corollary 3.4. For each integral ideal $\mathfrak{a}$ of $K$ and principal congruence subgroup $\Gamma_{K}(\mathfrak{a})$, we have

$$
c_{2}(Y(\mathfrak{a}))=\left[\Gamma_{K}: \Gamma_{K}(\mathfrak{a})\right] \frac{|D|^{5 / 2}}{32 \pi^{3}} L\left(3, \chi_{D}\right) .
$$

Remark 3.5. The above volume formula can be seen as a special case of a general formula developed by G. Prasad, expressing the volume of a quotient of a Lie group by an arithmetic subgroup, by values of $L$-functions. The reader can consult [Prasad and Yeung 2007] for the case of the unitary group corresponding to a hermitian form of signature $(2,1)$.

A similar arithmetic expression for the self-intersection number of the compactification divisor can be deduced using [Holzapfel 1980, Lemma 4.7, Lemma 4.8]:

Proposition 3.6. Let $h_{K}$ denote the ideal class number of $K$ and let $\eta_{K}$ and $\vartheta_{\mathfrak{a}}$ be defined as

$$
\begin{aligned}
& \vartheta_{\mathfrak{a}}=\min \{n \in \mathbb{N}|\mathfrak{a}| n \sqrt{-d}\}, \\
& \eta_{K}= \begin{cases}1 & \text { if } D=-4, \\
\frac{1}{6} & \text { if } D=-3 \\
2 & \text { if } D \neq-4, D \equiv 0 \bmod 4 \\
\frac{1}{2} & \text { if } D \neq-3, D \equiv 1 \bmod 4\end{cases}
\end{aligned}
$$

Then the following formula for the self-intersection number $\left(T_{\Gamma_{K}(\mathfrak{a})} \cdot T_{\Gamma_{K}(\mathfrak{a})}\right)$ holds:

$$
\left(T_{\Gamma_{K}(\mathfrak{a})} \cdot T_{\Gamma_{K}(\mathfrak{a})}\right)=-\frac{h_{K} \eta_{K}}{\vartheta_{\mathfrak{a}}^{2}}\left[\Gamma_{K}: \Gamma_{K}(\mathfrak{a})\right]
$$

Values of Dirichlet L-functions at integers. In order to get concrete values of Chern numbers of Picard modular surfaces, we need to know the value $L\left(3, \chi_{D}\right)$ explicitly. Let us briefly discuss the well-known method for the computation of this number. 
We first recall the functional equation for Dirichlet $L$-functions (see, for instance, [Washington 1982], Chapter 4, in particular Theorem 4.2). This equation implies

$$
L\left(3, \chi_{D}\right)=-\frac{2 \pi^{3}}{|D|^{5 / 2}} L\left(-2, \chi_{D}\right)=\frac{2 \pi^{3}}{3|D|^{5 / 2}} B_{3, \chi_{D}} .
$$

There, $B_{n, \chi_{D}}$ denotes the $n$-th generalized Bernoulli number associated with $\chi_{D}$ (see [Washington 1982, Chapter 4] for the definition). The generalized Bernoulli numbers can be computed easily by the following formula, which, for instance, can be found in [Washington 1982, Proposition 4.2]:

$$
B_{n, \chi_{D}}=|D|^{n-1} \sum_{k=1}^{|D|} \chi_{D}(k) B_{n}\left(\frac{k}{|D|}\right) .
$$

In the above formula, $B_{n}(X)$ denotes the $n$-th Bernoulli polynomial, which can be defined as

$$
B_{n}(X)=\sum_{k=0}^{n}\left(\begin{array}{l}
n \\
k
\end{array}\right) B_{k} X^{n-k}
$$

with $B_{k}$ denoting the usual $k$-th Bernoulli number. It is now easy to provide a list of values $L\left(3, \chi_{D}\right)$, knowing $B_{3}(X)=\frac{1}{2} X-\frac{3}{2} X^{2}+X^{3}$. Here we give the first values of $B_{3, \chi_{D}}$ :

\begin{tabular}{c|cccccccccccc}
$|D|$ & 3 & 4 & 7 & 8 & 11 & 15 & 19 & 20 & 23 & 24 & 31 & 35 \\
\hline$B_{3, \chi_{D}}$ & $2 / 3$ & $3 / 2$ & $48 / 7$ & 9 & 18 & 48 & 66 & 90 & 144 & 138 & 288 & 108
\end{tabular}

\section{Applications}

In this section we discuss some applications of the explicit formulas for the Chern invariants of compactified ball quotients by principal congruence subgroups.

\section{Classification.}

Theorem 4.1. Let $\mathfrak{a}$ be an integral ideal of $K=\mathbb{Q}(\sqrt{-d})$ such that $N(\mathfrak{a})>3$ and $\mathfrak{a} \cap \mathbb{Z}$ and the prime 2 are coprime. Then $\tilde{Y}(\mathfrak{a})$, the smooth compactification of $\Gamma_{K}(\mathfrak{a}) \backslash \mathbb{B}$, is a surface of general type, with the (additional) possible exception for $K=\mathbb{Q}(\sqrt{-7})$ and $\mathfrak{a} \cap \mathbb{Z}$ ramified in $K$.

Proof. First we note that $\tilde{Y}(\mathfrak{a})$ is a smooth and compact algebraic surface, since $\Gamma_{K}(\mathfrak{a})$ is neat by Lemma 2.6 (see also Theorem 3.1). In order to show that $\tilde{Y}(\mathfrak{a})$ is of general type it is sufficient to check that $c_{1}^{2}(\tilde{Y}(\mathfrak{a}))>9$. This follows from the classification theory of algebraic surfaces. It is also sufficient to check the inequality only for the surfaces $\tilde{Y}(\mathfrak{p})$, where $\mathfrak{p}$ is a prime ideal, since the Kodaira dimension 
can not decrease by the passage to a finite covering. Hence, if $\tilde{Y}(\mathfrak{p})$ is of general type, then so is $\tilde{Y}(\mathfrak{a})$ for any ideal $\mathfrak{a}$ such that $\mathfrak{p} \mid \mathfrak{a}$.

From Proposition 3.2, Corollary 3.4, and Proposition 3.6, we obtain the following formula for $c_{1}^{2}(\tilde{Y}(\mathfrak{p}))$ :

$$
c_{1}^{2}(\tilde{Y}(\mathfrak{p}))=\left[\Gamma_{K}: \Gamma_{K}(\mathfrak{a})\right]\left(\frac{3|D|^{5 / 2}}{32 \pi^{3}} L\left(3, \chi_{D}\right)-\frac{h_{K} \eta_{K}}{\vartheta_{\mathfrak{p}}^{2}}\right) .
$$

Looking at the index formula in Theorem 2.5, we see that $\left[\Gamma_{K}: \Gamma_{K}(\mathfrak{p})\right]>9$ if $N(\mathfrak{p})>3$ and $\mathfrak{p} \cap \mathbb{Z} \neq(2)$. Thus, it suffices to show that

$$
\frac{3|D|^{5 / 2}}{32 \pi^{3}} L\left(3, \chi_{D}\right)-\frac{h_{K} \eta_{K}}{\vartheta_{\mathfrak{p}}^{2}} \geq 1 \text {. }
$$

First we show that the inequality (4-2) holds if $|D|>35$.

Recall first the analytic class number formula

$$
h_{K}=\frac{\mu_{K} \sqrt{-D}}{2 \pi} L\left(1, \chi_{D}\right)=-\left(\mu_{K} / 4\right) B_{1, \chi_{D}},
$$

where $\mu_{K}$ denotes the number of roots of unity contained in $K$. Using the facts on generalized Bernoulli numbers summarized on page 334, and in particular the formula (3-1), we get a trivial bound $h_{K} \leq|D| / 4$ which holds for $|D| \neq 3$, 4. On the other hand, comparing the Euler factors, the value $L\left(3, \chi_{D}\right)$ is estimated below by a known value of the Riemann zeta function, namely, $L\left(3, \chi_{D}\right)>1 / \sqrt{\zeta_{\mathbb{Q}}(2)}=\sqrt{6} / \pi$. More precisely, one shows that $\left(1-p^{-2}\right)\left(1-\chi_{D}(p) p^{-3}\right)^{2}<1$ for all primes, from which the lower bound follows. Noting additionally that always $\eta_{K} \leq 2$ and $\vartheta_{\mathfrak{p}} \geq 1$, the inequality (4-2) will follow from the inequality

$$
|D|^{5 / 2}>\frac{16 \pi^{4}}{3 \sqrt{6}}(2+|D|) .
$$

This inequality holds for $|D|>35$.

For discriminants $D$ with $|D| \leq 35$ we have to check the inequality $c_{1}^{2}(\tilde{Y}(\mathfrak{p}))>9$ case by case. For this, one first uses the functional equation to reformulate (4-1) as

$$
c_{1}^{2}(\tilde{Y}(\mathfrak{p}))=\left[\Gamma_{K}: \Gamma_{K}(\mathfrak{a})\right]\left(\frac{3}{16} B_{3, \chi_{D}}-\frac{h_{K} \eta_{K}}{\vartheta_{\mathfrak{p}}^{2}}\right)
$$

and then uses the exact values $B_{3, \chi_{D}}$ from the table on page 335 . For instance, let us consider the case $D=-35$. With $h_{K}=2, \eta_{K}=\frac{1}{2}$ and $B_{3}, \chi_{-35}=108$ we have $c_{1}^{2}=\left[\Gamma_{K}: \Gamma_{K}(\mathfrak{p})\right]\left(\frac{81}{4}-1 / \vartheta_{\mathfrak{p}}^{2}\right) \geq \frac{81}{4}\left[\Gamma_{K}: \Gamma_{K}(\mathfrak{p})\right]>9$. The other cases are treated in the same manner. The cases $|D|=-3,-4,-7,-8$ need to be considered more carefully, since in these situations $c_{1}^{2}$ can be negative.

$D=-8$. Let us, for brevity, write $I_{\mathfrak{p}}$ instead of $\left[\Gamma_{K}: \Gamma_{K}(\mathfrak{p})\right]$. For the self-intersection we have $c_{1}^{2}=I_{\mathfrak{p}}\left\{\frac{27}{48}-2 / \vartheta_{\mathfrak{p}}^{2}\right\}$. Now, it is easy to see that $c_{1}^{2}<0$ if and only if 
$\vartheta_{\mathfrak{p}}=1$, which is exactly the case when $p=\mathfrak{p} \cap \mathbb{Z}$ is ramified in $K=\mathbb{Q}(\sqrt{-2})$. From this it follows that $p=2$, and this case is excluded. Otherwise, $c_{1}^{2}>9$ if $I_{\mathfrak{p}}>144$. This is the case for $N(\mathfrak{p})>3$ and $p$ unramified, as the index formula shows.

$D=-7$. Here we have $c_{1}^{2}=I_{\mathfrak{p}}\left\{\frac{3}{7}-\frac{1}{2} \vartheta_{\mathfrak{p}}^{2}\right\}$. This expression is negative if and only if $\vartheta_{\mathfrak{p}}=1$, which means that $p=\mathfrak{p} \cap \mathbb{Z}$ is ramified. This is the exception we have to exclude. Indeed, for $\mathfrak{p}=(\sqrt{-7})$, we have $c_{2}=48$ and $c_{1}^{2}=-24$. In other cases $c_{1}^{2}>9$.

$D=-4$. Here we get a negative $c_{1}^{2}$ for $\vartheta_{\mathfrak{p}}=1$ or 2 . But $\vartheta_{\mathfrak{p}}=1$ is only possible for $\mathfrak{p}=(1)$ and $\vartheta_{\mathfrak{p}}=2$ is only possible for those $\mathfrak{p}$ which contain the prime 2 .

$D=-3$. In this case $c_{1}^{2}<0$ is only possible for a prime ideal $\mathfrak{p}$ such that $p$ is ramified in $K$. But then $N(\mathfrak{p})=3$, a case which is excluded.

Remark 4.2. Let us remark that the above proof works for any neat Picard modular group. Let, for instance, $K$ be the imaginary quadratic field of discriminant $D=-24$ and $\Gamma$ be the congruence subgroup $\Gamma_{K}(2 \sqrt{D})$. This group is neat (see [Dimitrov and Ramakrishnan 2015, Proposition 2.6]). The group $\Gamma$ is a subgroup of $\Gamma_{K}\left(\mathfrak{p}_{3}\right)$, where $\mathfrak{p}_{3}$ is the prime ideal over the rational prime $p=3$. By Lemma $2.4,\left[\Gamma_{K}: \Gamma\right]>24$. The arguments of the proof of Theorem 4.1 then lead to $c_{1}^{2}(Y(2 \sqrt{D}))>9$ and we conclude that $Y(2 \sqrt{D})$ is of general type.

The invariants of $\tilde{Y}(\mathfrak{a})$ are in general very large due to the fact that the index, which grows very fast with the norm of the ideal, dominates the expressions. However, the surfaces $\tilde{Y}(\mathfrak{a})$ are interesting from the point of view of surface geography, since the pairs $\left(c_{1}^{2}(\tilde{Y}(\mathfrak{a})), c_{2}(\tilde{Y}(\mathfrak{a}))\right)$ are located in an interesting region of the $\left(c_{1}^{2}, c_{2}\right)$-plane, namely the ratio $c_{1}^{2} / c_{2}$ is close to 3 (see [Holzapfel 1980], and the first part of [Hunt 1989], for a detailed discussion on surface geography). More precisely, the ratio $c_{1}^{2}(\tilde{Y}(\mathfrak{a})) / c_{2}(\tilde{Y}(\mathfrak{a}))$ tends to 3 when either the norm $N(\mathfrak{a})$ or the discriminant $|D|$ tends to infinity (see also [Holzapfel 1980]). This is easily seen since

$$
\frac{c_{1}^{2}(\tilde{Y}(\mathfrak{a}))}{c_{2}(\tilde{Y}(\mathfrak{a}))}=3-\frac{h_{K} \eta_{K} / \vartheta_{\mathfrak{a}}^{2}}{\frac{|D|^{5 / 2}}{32 \pi^{3}} L\left(3, \chi_{D}\right)} .
$$

Now, as $\vartheta_{\mathfrak{a}}^{2} \rightarrow \infty$ for $N(\mathfrak{a}) \rightarrow \infty$, we see that for a fixed $D$ the ratio tends to 3 . Let us, on the other hand, fix a but let $D$ vary. We first observe, as $L\left(3, \chi_{D}\right)$ is bounded by the value of the Riemann zeta function at $s=3$, namely $\zeta(3)^{-1}<$ $L\left(3, \chi_{D}\right)<\zeta(3)$, that there are constants $C$ and $C^{\prime}$ not depending on $D$ such that

$$
C^{\prime} \frac{h_{K}}{|D|^{5 / 2}} \geq \frac{\frac{h_{K} \eta_{K}}{\vartheta_{\mathfrak{a}}^{2}}}{\frac{|D|^{5 / 2}}{32 \pi^{3}} L\left(3, \chi_{D}\right)} \geq C \frac{h_{K}}{|D|^{5 / 2}} .
$$


There are well-known bounds for the class number of an imaginary quadratic field, namely, for any $\epsilon>0$, there are constants $C_{1}=C_{1}(\epsilon)$ and $C_{2}=C_{2}(\epsilon)$ such that

$$
C_{1}|D|^{1 / 2+\epsilon}>h_{K}>C_{2}|D|^{1 / 2-\epsilon} \text {. }
$$

Bringing these bounds into (4-5), we see that the expression $\frac{h_{K} \eta_{K}}{\vartheta_{a}^{2}} / \frac{|D|^{5 / 2}}{32 \pi^{3}} L\left(3, \chi_{D}\right)$ tends to zero as $|D| \rightarrow \infty$. Hence

$$
\lim _{|D| \rightarrow \infty} \frac{c_{1}^{2}(\tilde{Y}(\mathfrak{a}))}{c_{2}(\tilde{Y}(\mathfrak{a}))}=3 .
$$

Already for small discriminants and norms, the Chern number ratio is very close to 3 (see [Holzapfel 1980], Table 1).

Dimension formulas. Another application of the above explicit formulas for the Chern numbers of Picard modular surfaces is an explicit formula for the dimensions of spaces of cusp forms relative to a Picard modular group $\Gamma$.

A holomorphic function $f$ on the ball is called a cusp form of weight $k$ with respect to $\Gamma$ if it satisfies the following conditions:

- For all $\gamma \in \Gamma$ and all $z \in \mathbb{B}, f(\gamma z)=j(\gamma, z)^{k} f(z)$, where $j(\gamma, z)$ denotes the determinant of the Jacobian matrix corresponding to the holomorphic map $\gamma: \mathbb{B} \rightarrow \mathbb{B}$ at the point $z$.

- The function $f(z)$ vanishes at cusps of $\Gamma$, i.e., it vanishes on $\partial_{\Gamma} \mathbb{B}=\Gamma \backslash \partial_{\mathbb{Q}} \mathbb{B}$.

Let $\Gamma \subset \Gamma_{K}$ be a neat Picard modular group. In the following, $S_{k}(\Gamma)$ will denote the space of cusp forms of weight $k$ with respect to $\Gamma$. By a result of Hemperly [1972], the cusp forms of weight $k$ can be interpreted as the sections of a certain line bundle $\mathbb{L}^{(k)}$ on the smooth compactification $\tilde{Y}(\Gamma)$. Thus, $\operatorname{dim} S_{k}(\Gamma)=$ $\operatorname{dim} H^{0}\left(\tilde{Y}(\Gamma), \mathbb{L}^{(k)}\right)$. As an application of the Riemann-Roch theorem and the Kodaira vanishing theorem, one gets the following formula for $\operatorname{dim} S_{k}(\Gamma)$ :

Theorem 4.3 (see [Holzapfel 1998b]). Let $\Gamma$ be a neat Picard modular group and $k \geq 2$. Then

$$
\operatorname{dim} S_{k}(\Gamma)=\left[\Gamma_{K}: \Gamma\right]\left(\frac{1}{6}(9 k(k-1)+2) c_{2}(Y(\Gamma))+\frac{1}{12}\left(T_{\Gamma} \cdot T_{\Gamma}\right)\right) .
$$

Now it is clear that we have an explicit formula for the dimensions of spaces of cusp forms of weight $k \geq 2$ which are automorphic with respect to a neat principal congruence subgroup $\Gamma_{K}(\mathfrak{a})$, namely,

$$
S_{k}\left(\Gamma_{K}(\mathfrak{a})\right)=\frac{\left[\Gamma_{K}: \Gamma_{K}(\mathfrak{a})\right]}{6}\left((9 k(k-1)+2) \frac{|D|^{5 / 2}}{32 \pi^{3}} L\left(3, \chi_{D}\right)-\frac{h_{K} \eta_{K}}{2 \vartheta_{\mathfrak{a}}^{2}}\right) .
$$

These dimensions are very large in general, even in the case of small discriminants and small norms. 


\section{References}

[Baeza 1973] R. Baeza, "Eine Zerlegung der unitären Gruppe über lokalen Ringen", Arch. Math. (Basel) 24 (1973), 144-157. MR Zbl

[Bass 1964] H. Bass, “K-theory and stable algebra”, Inst. Hautes Études Sci. Publ. Math. 22 (1964), 5-60. MR

[Borel and Harish-Chandra 1962] A. Borel and Harish-Chandra, "Arithmetic subgroups of algebraic groups", Ann. of Math. (2) 75 (1962), 485-535. MR Zbl

[Dimitrov and Ramakrishnan 2015] M. Dimitrov and D. Ramakrishnan, "Arithmetic quotients of the complex ball and a conjecture of Lang", Doc. Math. 20 (2015), 1185-1205. MR Zbl

[Hemperly 1972] J. C. Hemperly, "The parabolic contribution to the number of linearly independent automorphic forms on a certain bounded domain", Amer. J. Math. 94 (1972), 1078-1100. MR Zbl

[Holzapfel 1980] R.-P. Holzapfel, "A class of minimal surfaces in the unknown region of surface geography”, Math. Nachr. 98 (1980), 211-232. MR Zbl

[Holzapfel 1998a] R.-P. Holzapfel, Ball and surface arithmetics, Aspects of Mathematics E29, Friedr. Vieweg \& Sohn, Braunschweig, 1998. MR Zbl

[Holzapfel 1998b] R.-P. Holzapfel, "Zeta dimension formula for Picard modular cusp forms of neat natural congruence subgroups”, Abh. Math. Sem. Univ. Hamburg 68 (1998), 169-192. MR Zbl

[Hunt 1989] B. Hunt, "Complex manifold geography in dimension 2 and 3", J. Differential Geom. 30:1 (1989), 51-153. MR Zbl

[Lang 1970] S. Lang, Algebraic number theory, Addison-Wesley, Reading, MA, 1970. MR Zbl

[Prasad and Yeung 2007] G. Prasad and S.-K. Yeung, "Fake projective planes", Invent. Math. 168:2 (2007), 321-370. MR Zbl

[Washington 1982] L. C. Washington, Introduction to cyclotomic fields, Graduate Texts in Mathematics 83, Springer, New York, 1982. MR Zbl

Received November 17, 2015. Revised January 19, 2016.

AMIR DŽAMBIĆ

MATHEMATISCHES SEMinAR

CHRISTIAN-ALBRECHTS-UNIVERSITÄT ZU KIEL

LUDEWIG-MEYN-STRASSE 4

24098 KIEL

GERMANY

dzambic@math.uni-kiel.de 


\title{
PACIFIC JOURNAL OF MATHEMATICS
}

Founded in 1951 by E. F. Beckenbach (1906-1982) and F. Wolf (1904-1989)

$$
\text { msp.org/pjm }
$$

\section{EDITORS}

\author{
Don Blasius (Managing Editor) \\ Department of Mathematics \\ University of California \\ Los Angeles, CA 90095-1555 \\ blasius@math.ucla.edu
}

\author{
Paul Balmer \\ Department of Mathematics \\ University of California \\ Los Angeles, CA 90095-1555 \\ balmer@math.ucla.edu \\ Robert Finn \\ Department of Mathematics \\ Stanford University \\ Stanford, CA 94305-2125 \\ finn@math.stanford.edu \\ Sorin Popa \\ Department of Mathematics \\ University of California \\ Los Angeles, CA 90095-1555 \\ popa@math.ucla.edu
}

\author{
Vyjayanthi Chari \\ Department of Mathematics \\ University of California \\ Riverside, CA 92521-0135 \\ chari@math.ucr.edu \\ Kefeng Liu \\ Department of Mathematics \\ University of California \\ Los Angeles, CA 90095-1555 \\ liu@math.ucla.edu \\ Igor Pak \\ Department of Mathematics \\ University of California \\ Los Angeles, CA 90095-1555 \\ pak.pjm@gmail.com \\ Paul Yang \\ Department of Mathematics \\ Princeton University \\ Princeton NJ 08544-1000 \\ yang@math.princeton.edu
}

\section{PRODUCTION}

Silvio Levy, Scientific Editor, production@msp.org

\section{SUPPORTING INSTITUTIONS}

ACADEMIA SINICA, TAIPEI

CALIFORNIA INST. OF TECHNOLOGY

STANFORD UNIVERSITY

UNIV. OF BRITISH COLUMBIA

UNIV. OF CALIFORNIA, BERKELEY

UNIV. OF CALIFORNIA, DAVIS

UNIV. OF CALIFORNIA, LOS ANGELES

UNIV. OF CALIFORNIA, RIVERSIDE

UNIV. OF CALIFORNIA, SAN DIEGO

UNIV. OF CALIF., SANTA BARBARA
KEIO UNIVERSITY

MATH. SCIENCES RESEARCH INSTITUTE

NEW MEXICO STATE UNIV.

OREGON STATE UNIV.
Daryl Cooper

Department of Mathematics

University of California

Santa Barbara, CA 93106-3080 cooper@math.ucsb.edu

Jiang-Hua Lu

Department of Mathematics

The University of Hong Kong

Pokfulam Rd., Hong Kong

jhlu@maths.hku.hk

$$
\text { Jie Qing }
$$

Department of Mathematics

University of California

Santa Cruz, CA 95064

qing@ cats.ucsc.edu

\author{
UNIV. OF CALIF., SANTA CRUZ \\ UNIV. OF MONTANA \\ UNIV. OF OREGON \\ UNIV. OF SOUTHERN CALIFORNIA \\ UNIV. OF UTAH \\ UNIV. OF WASHINGTON \\ WASHINGTON STATE UNIVERSITY
}

These supporting institutions contribute to the cost of publication of this Journal, but they are not owners or publishers and have no responsibility for its contents or policies.

See inside back cover or msp.org/pjm for submission instructions.

The subscription price for 2016 is US $\$ 440 /$ year for the electronic version, and \$600/year for print and electronic.

Subscriptions, requests for back issues and changes of subscriber address should be sent to Pacific Journal of Mathematics, P.O. Box 4163, Berkeley, CA 94704-0163, U.S.A. The Pacific Journal of Mathematics is indexed by Mathematical Reviews, Zentralblatt MATH, PASCAL CNRS Index, Referativnyi Zhurnal, Current Mathematical Publications and Web of Knowledge (Science Citation Index).

The Pacific Journal of Mathematics (ISSN 0030-8730) at the University of California, c/o Department of Mathematics, 798 Evans Hall \#3840, Berkeley, CA 94720-3840, is published twelve times a year. Periodical rate postage paid at Berkeley, CA 94704, and additional mailing offices. POSTMASTER: send address changes to Pacific Journal of Mathematics, P.O. Box 4163, Berkeley, CA 94704-0163.

PJM peer review and production are managed by EditFLOW ${ }^{\circledR}$ from Mathematical Sciences Publishers.

PUBLISHED BY

\section{I. mathematical sciences publishers}

nonprofit scientific publishing

http://msp.org/

(C) 2016 Mathematical Sciences Publishers 


\section{PACIFIC JOURNAL OF MATHEMATICS}

Volume $283 \quad$ No. $2 \quad$ August 2016

The fundamental theorem of tropical differential algebraic geometry

FUENSANTA AROCA, CRISTHIAN GARAY and ZEINAB TOGHANI

A simple solution to the word problem for virtual braid groups

271

PaOlo Bellingeri, Bruno A. Cisneros de la CruZ and Luis

PARIS

Completely contractive projections on operator algebras

DAVID P. BLECHER and MATTHEW NEAL

Invariants of some compactified Picard modular surfaces and applications

AMIR DŽAMBIĆ

Radial limits of bounded nonparametric prescribed mean curvature surfaces

MOZHGAN (NORA) ENTEKHABI and KIRK E. LANCASTER

A remark on the Noetherian property of power series rings

BYUNG GYUN KANG and PHAN THANH TOAN

Curves with prescribed intersection with boundary divisors in moduli spaces of curves

\section{XIAO-LEI LIU}

Virtual rational Betti numbers of nilpotent-by-abelian groups

BEHROOZ MiRZAII and FATEMEH Y. MOKARI

A planar Sobolev extension theorem for piecewise linear homeomorphisms 405

EMANUELA RADICI

A combinatorial approach to Voiculescu's bi-free partial transforms

PAUL SKOUFRANIS

Vector bundle valued differential forms on $\mathbb{N} Q$-manifolds

LUCA VitAGLiANO

Discriminants and the monoid of quadratic rings 